\title{
Light sheet flow control technology research
}

\author{
Yueming Yang Jinbo Yao Qingyu Yang Xuewei Liu \\ Air Force Aviation University \\ Changchun,china \\ ccyym1@126.com
}

\begin{abstract}
This article by the understanding of the basic principles of flow visualization, $0.75 \mathrm{~m} \times \mathbf{0 . 7 5 \mathrm { m }}$ backflow wind tunnel since the inquiry light sheet flow display device,Observe the liquidity situation of the aircraft model in the low-speed state achieved good results.
\end{abstract}

\section{Keywords-Flow visualization;Eddy;Light sheet;Lens}

\section{OVERVIEW OF FLOW VISUALIZATION}

Process visualization flow visualization technology task is to make the fluid transport phenomena. Through a variety of flow visualization experiments to understand the complex flow phenomena, to explore the physical mechanisms for people to discover new flow phenomena, an important means to create new concepts and provide the basis for the physical model, but also to solve practical engineering problems.

Flow visualization technology development with the development of fluid mechanics, has been 100 years of history. It can be said that the fluid dynamics in the development process, every major discovery in the academic and applied to the engineering of the actual almost from the observed flow phenomena began. Reynolds transition experiments; Mach shock wave phenomenon observed; Prantl metal powder tracer particles, a stream along the flat spectrum, proposed the concept of the boundary layer on the sink; V.Karman cylinder around flow observation and Karman Vortex street proposed; Detached eddy research; turbulent coherent structures in found type of proposed research and separation of the flow stream; separation at high angles of attack, and so on, are all based on flow visualization and measurement of out of body based on the results. In-depth analysis of the flow phenomena is to establish and validate new concepts, found the key to the new flow law.

Modern hydrodynamics and aerodynamics development as well as the separation of the flow pattern in the research and development of a new generation of aircraft, for people to complex flows (for example, separated flow, vortex flow, turbulence, unsteady flow, etc.) raised new issues including its mechanism and applied research. These complex flow generally is three-dimensional, unsteady, aperiodic pieces, coherent, and their behavior changes over time and space, or has a complex spatial structure, or the flow of non-steady, and often both. It should be pointed out that, since the capture of these complex flow phenomena and flow mechanism and the law is not enough research, including one of the important reasons is the lack of a means of display and measurement of complex flow. Over the last decade, due to the urgent need of the engineering practice and modern optics, laser technology, computer technology, electronic technology,

information processing technology development, has brought vigor and vitality for flow visualization and measurement technology, especially in the display space flow and flow capacity of the internal structure of quantitative extraction as well as the flow of information and analysis at four aspects have made great progress, achieved a major breakthrough in the three-dimensional, unsteady complex flow quantitative measurement expect in the near future.

\section{FLOW VISUALIZATION AND MEASUREMENT TECHNOLOGY}

The range flow visualization and measurement methods, usually traditional flow visualization and measurement methods; computer-aided flow visualization and measurement methods (ie, flow visualization and measurement and computer image processing method of combining).

Taking into account the complex flow of aircraft development and other projects recognize the urgent need for and the huge advances in laser technology, computer technology,information processing technology,the produced particle image velocimetry(PIV),laser-induced fluorescence(LIF),laser molecular velocimetry(LMV), pressure-sensitive coating manometry (PSP) is a representative of the new generation of mobile measurement technology, they both the qualitative display and quantitative measurement ability, and some unsteady complex flow space transient structure display and measurement, so that the study of complex flow breakthrough progress.

A The basic principle of the light sheet flow visualization techniques

Light sheet flow visualization technique is a way of lighting in the flow visualization of the space and lighting technology, it is a cross-sectional flow of the flow field, flow visualization experiments need to illuminate the display space of mobile technology in a variety of tracer particles space to three-dimensional flow field image of the cross-sectional flow stream spectrum. Multiple sections of the complex three-dimensional flow phenomena chip optical technology on display direct shooting and recording. 


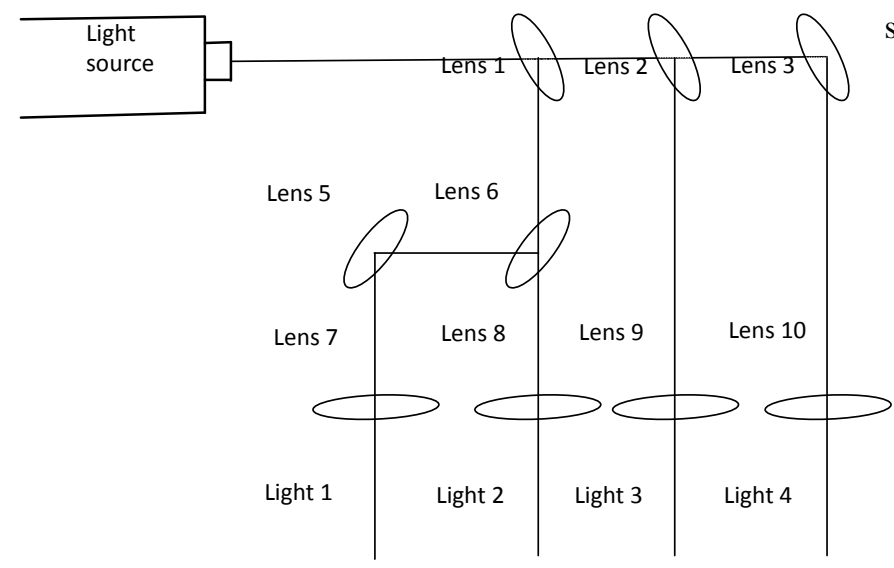

Figure 1 optical device optical path diagram

Certain flow field exists in a specific area, its flow rate and its velocity of the flow field around the obvious differences, If it is invested in the region upstream of the tracer particles, illuminated with a bright light in the region and the flow field around a cross-section, When tracer particles flow through the cross-sectional, illuminated light scattering in all directions. Sheet illuminated by the light of the region always has a certain thickness, the thickness of the light by the sheet decided, typically tenths of a millimeter to a few millimeters. Tracer particles flowing through the cross section of the sheet illuminated by the light, the lower speed of tracer particles in the light sheet area to a longer residence time, and the short residence time of the high speed of the tracer particles, and therefore in a small time interval high number of tracer particles in the low speed flow field in the cross section of the light sheet density greater than the speed of the tracer particle density, scattering light is strong, while the latter is weak. So that flow through the light sheet the tracer particles sectional groups scattering light intensity difference between the flow rate difference can be displayed on each cross-section of the flow field, which is the principle of light sheet flow visualization.

\section{$B$ Light sheet display device design}

Optical fiber-chip optical device is installed on a low-speed wind tunnel test section $0.75 \mathrm{~m} \times 0.75 \mathrm{~m}$ backflow basic experimental apparatus comprising: a laser light sheet device tracer particle generator, optical scanners, data processing and memory . The device has a highly integrated, flexible adjustment, display function, low cost and features

Light source with a solid emerald laser power of $1 \mathrm{~W}$.

Light sheet device shown in Figure 2, the device light sheet prismatic the spectrophotometric piece 4 , every piece of the light power $0.25 \mathrm{w}$, basically covers the experimental device, also according to the need to move around, to illuminate all Experimental devices. The lens 1 and the lens 6 is a convex lens, a lens 7 to the lens 10 is a cylindrical lens, a key member for forming sheets of light.

Tracer particle generator, stage smoke generator.

Manual shift measured frame using the advantages of simple structure, moving arbitrarily accurate, low cost.

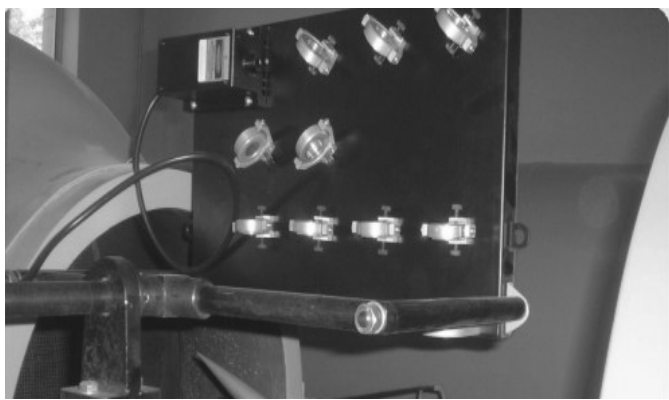

Fig2 Light sheet device

\section{CONCLUSION}

Through nearly three years of experiments, the Light sheet, test device Light sheet and tracer particles control to good effect, high integration, flexible adjustment, strong function, low cost.
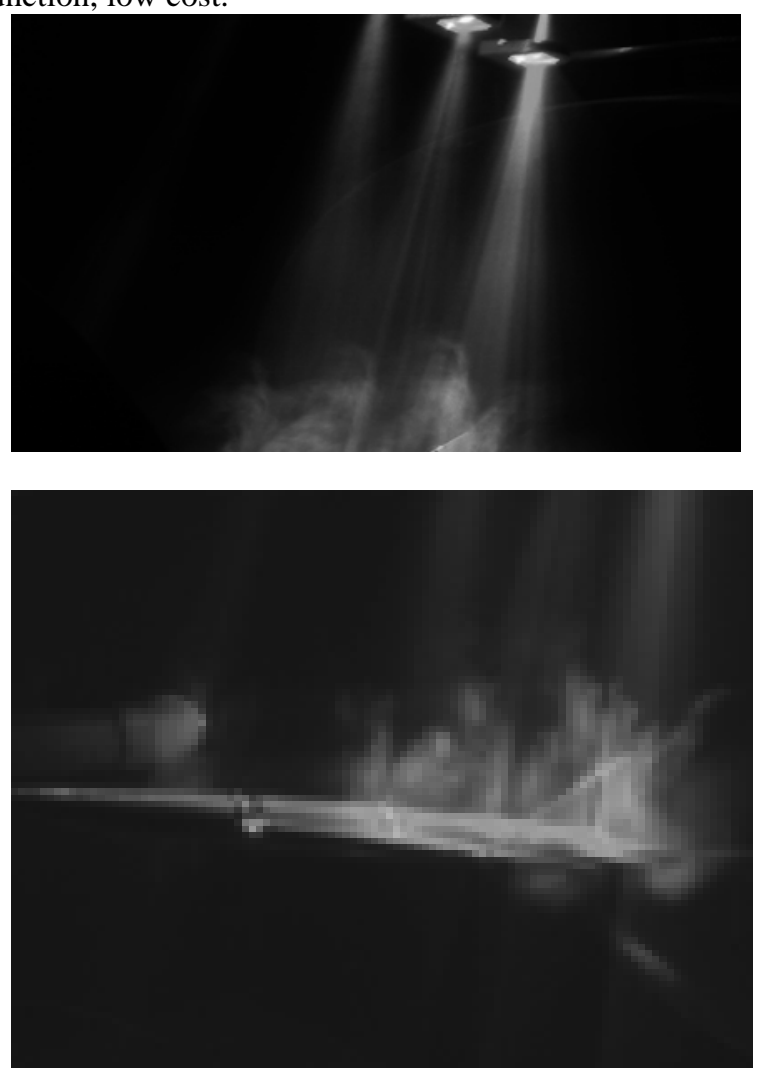

Fig 4 light test - vortex breakdown

Good test results, showing the overall structure of the vortex core morphology, including the generation, the development process of the vortex, the vortex core toward changes in the vortex core diameter, the breaking point of the vortex and the breakdown of the situation. 


\section{ACKNOWLEDGINENT}

This paper supported by National Natural Science Foundation China (Grant NO:61172126/F010410)

\section{REFERENCES}

[1] Fan Jiechuan Modern flow display technology [M] Beijing: National Defense Industry Press, 2002.1

[2] Li guichun Compile. wind tunnel tests optical measurement method [M] Beijing: National Defense Industry Press, 2008.5.

[3] Wind tunnel test optical measurement method. [M]. Beijing : Beijing Institute of Aeronautics Press. 1985.10

[4] Wuronglin. Wind tunnel design principles [M]. Beijing : Beijing Institute of Aeronautics Press. 1985.10

[5] Bao guohua. Wind tunnel special experiment [M]. Xian : Northwestern Polytechnical University Press. 1990.5 